\title{
EL TRABAJO EN EQUIPO: UNA ALTERNATIVA PARA EL CAMBIO DE LA CULTURA INSTITUCIONAL
}

\author{
M.Sc. Monika Flochová Luptáková
}

Los sujetos que confornan una institución deben organizarse para trabajar en equipos. En lo intermo de esas agrupaciones surgen lideres, jerarquias, se asignan tareas, se cumplen evaluaciones. En otras palabras, el grupo construye una cultura que incide directamente en los procesos y productos de su trabajo. La autora nos presenta varias alternativas para obtener mayor provecho de las labores grupales. Por lo tanto, se trata de un articulo de utilidad para los docentes de los diversos niveles, desde el preescolar hasta la educación superior, asi como a administradores de instituciones públicas y privadas.
The persons that make up an institution should organize themselves for teamwork. Within these institutions, leaders, hierarchies, and tasks arise and evaluations take place. In short, the group builds an organizational culture that has a direct effect on the processes and products of their work. The author presents several alternatives to better capitalize on teamwork. This article is very useful for teachers working in the different levels of the educational system, from preschool to higher education, and for administrators of public and private institutions.

* Licenciada en Psicología por la Universidad Comenio, Checosloaquia y Máster en Estudios Grupales por Universidad para la Cooperación Intemacional en Costa Rica. Docente de la Escuela de Psicología de la Facultad de Ciencias Sociales de la Universidad Nacional. . 
Por lo tanto, al estar en un grupo, es importante la escucha del otro, la empatía con el otro, para captar la realidad del otro
El presente artículo pretende ser útil para todas aquellas personas que realizan trabajos con y en grupos, ya sean laborales, de formación, terapéuticos o de autoayuda y desean conocer acerca de los factores que influyen en la dinámica grupal. En este sentido Barreiro ( 1993, p.1) afirma "el trabajo grupal es un instrumento importante para mejorar las instituciones, para promover mejoras en la educación, en salud y en otros ámbitos afines, y para estimular el crecimiento personal."

Shapira (1991, p. 66) señala que "el denominador común que las instituciones tienen para sus empleados se basa en identificarlas con su función materna" ya que las instituciones representan simbólicamente la madre, la cual los contiene, les disipa sus ansiedades y les da sentido de pertenencia y de reconocimiento (Podcaminsky, Beausset, 1999), aspectos de suma importancia para los seres humanos. Lo mismo ocurre cuando se forma, constituye y comienza a funcionar un grupo, que puede llegar a ser un equipo de trabajo. Para ello es importante conocer los elementos que forman parte de la estructura grupal.

Estos tienen que ver con la identificación de la tarea, con la identificación de quienes participan entre sí, con el contexto institucional, y con los roles que asumen las personas que integran un grupo en relación con la tarea por realizar. Me refiero a los aspectos que forman parte del proceso grupal. En un equipo de trabajo hay que tomar en cuenta otros elementos que interfieren en el proceso grupal. Se conocen como organizadores del grupo. Me refiero también a la fantasía individual, a los miedos, al vínculo primario y la imagen del líder que cada integrante trae al grupo.

El presente marco conceptual es integrativo, ya que une los aportes al campo grupal, que hacen diferentes especiali-dades de psicología tales como el psicoanálisis, el enfoque humanista, la psicología social, el enfoque de género y otras ciencias afines.

Es importante aclarar lo que entendemos por marco referencial y operativo. Pichón-Riviere, según Quiroga (1996: 1) lo define de la siguiente manera: "El esquema conceptual, referencial y operativo, llamado ECRO, es un conjunto organizado de nociones y conceptos generales, teóricos, que permite la aproximación instrumental al objeto particular concreto."

El ECRO y la didáctica que lo vehiculiza se fundamenta en el método dialéctico. El marco referencial y operativo nos permite hacer la lectura de una situación grupal, que ocurre dentro de un contexto ya sea a nivel institucional, nacional o internacional. 
Pichón-Riviere señala que el concepto de esquema, representa relaciones entre las ideas abstractas. Estas forman un sistema de ideas que tienen una organización conceptual donde "cada situación de aprendizaje sirve como el punto de referencia para comprender las distintas situaciones que se dan a nivel individual, grupal, institucional o comunitario" (Chaves, 1998: 1).

De esta manera se obtiene un instrumento teórico que permite hacer la lectura de una realidad. Quiroga (1996: 8) plantea que, la realidad "es un conjunto de hechos, de procesos, de fenómenos interconectados y relacionados entre sí". Por lo tanto, al estar en un grupo, es importante la escucha del otro, la empatía con el otro, para captar la realidad del otro, sin que eso implique emitir juicio de valor acerca de lo que otra persona explicita en su momento (Rud, 1994). La apertura de no juzgar abre la posibilidad para que quienes participan en un grupo externen sus opiniones, se sientan libres, confiados y alegres.

Agregar a la lectura de los fenómenos grupales la visión de género, significa comprender "que muchas cosas que creemos que son naturales, solamente porque nacimos hombres o nacimos mujeres, en realidad no lo son" (Lagarde, 1994: 4).

$\mathrm{Al}$ retomar aportes de varias disciplinas y teorías al trabajo grupal este se visualiza como "una herramienta de cambio y de transformación" (Barreiro, 1993: 2) que promueve el bienestar, reduce el malestar y mejora la calidad de vida de las personas en una institución. Por lo tanto es importante destacar en este campo los trabajos de algunos autores como Lewin, Rodgers, Moreno, Freud, Bleger, Fernández, Fumigalli, entre otros.

Según Barreiro (1993: 86) lo integrativo, señalado por Pichón-Riviere, lo representa "la integración de distintos aspectos de la persona, que muchas veces se encuentran escondidos y organizados en pares antinómicos (lo individual y lo grupal, lo intelectual y lo afectivo, el aprendizaje y la producción del conocimiento)".

De esta manera, la propuesta de integración representa la necesidad de construir una teoría que refleja "confluencia de aportes provenientes de distintos campos de conocimiento" (Barreiro, 1993: 87) y lo que conlleva una búsqueda interdisciplinaria de diferentes ciencias como la antropología, biología, sociología, pedagogía, filosofía entre otras y "supone la adopción de un encuadre epistemológico" (Barreiro, 1993: 87).

\section{Elementos de la estructura grupal}

\section{El agrupamiento y el grupo}

El análisis del campo de lo grupal requiere claridad conceptual para establecer la diferencia entre lo que es y que no es un grupo. Barreiro (1993: 13) 
entiende por agrupamiento "un conjunto de personas que están físicamente juntas, pero no interactúan entre sí o lo hacen de manera circunstancial".

Chibás (1992: 5) define grupo como un conjunto de personas que interactúan directamente entre sí, reunidas para alcanzar un objetivo común y que llegan a integrar una formación relativamente estable en el tiempo con una determinada estructura (roles y normas grupales) y procesos dinámicos internos". Otro referente de lo que es un grupo lo aporta Pichón-Riviere, (Bleger, 1961: 57) quien define grupo como "un conjunto restringido de personas que ligadas entre sí por constantes de tiempo y espacio, y articuladas por su mutua representación interna se proponen en forma explícita e implícita una tarea que constituye su finalidad, interactuando la adopción y adjudicación de roles".

\section{Tarea}

La tarea reúne al coordinador con su grupo y éste se organiza a su alrededor. En el abordaje de la tarea y en la búsqueda de soluciones, el grupo puede atravesar por cuatro fases en las cuales el elemento esencial en la comunicación es la escucha del otro, entenderlo y comprender su idea en relación con la tarea a la que está abocado el grupo. Es de suma importancia que, en la primera fase, un grupo defina su tarea y las normas básicas de su funcionamiento. Chibás (1992: 25), afirma que "ésta es una fase particularmente difícil, que exige un enorme esfuerzo del coordinador. En esta fase no es recomendable criticar ideas de ningún integrante del grupo".

La segunda fase, según el mismo autor, se conoce como fase de evaluación, durante la cual se escogen las mejores ideas.

En la tercera fase se desarrolla un plan de acción para poner en práctica las ideas antes seleccionadas y que - en un determinado momento y tiempo- se van a realizar.

En la cuarta fase se ejecuta el plan de acción elaborado anteriormente.

En relación con la tarea, se reconocen tres momentos que se presentan sucesivamente en cada situación.

El primer momento lo conforma la pre-tarea, durante la cual aparecen mecanismos de defensa que estructuran lo que se conoce "como resistencia al cambio" (Bauleo y De Brassi, 1990). Son movilizados por el miedo que opera como obstáculo. Se establece la distancia entre la realidad y lo deseado, surge tensión y se buscan salidas para lograr "un como si" de la tarea. En la pre-tarea se postergan cosas, se deja pasar el tiempo. Lo que se oculta es la dificultad de tolerar las tensiones relacionadas con el inicio y la terminación de la tarea. La postergación de la tarea genera entre los miembros del grupo frustración constante y aumento de la ansiedad. 
El segundo momento surge con el abordaje de la tarea y cuando se elaboran las ansiedades, se sale del estancamiento y el grupotiene que elaborar estrategias de intervención.

El tercer momento lo representa la elaboración del proyecto o cuando se llega a esclarecer la tarea, se logra el insight. Esto requiere coherencia entre el pensar, el sentiry la acción en una situación dentro de un contexto institucional.

\section{Coordinación}

En todo grupo sus integrantes empiezan a relacionarse entre sí y con su coordinadora o coordinador. La persona a cargo del grupo, con sus actitudes, propone un determinado modelo de vinculación, marca pautas de comunicación mediante gestos o con su léxico. Así, el que coordina va plasmando lo que Goffman llama una "definición de la situación" y se va estableciendo un determinado clima grupal (Barreiro, 1993). Cuando las pautas de comunicación desde la coordinación tienden a descalificar y enjuiciar, se instala en el grupo una matriz competitiva y el clima se llena de tensión. Por el contrario, si el coordinador o la coordinadora promueven confianza mutua y aceptación, se abandonan poco a poco las actitudes agresivas y defensivas y comienza a instalarse un apoyo mutuo que es la base de la matriz cooperativa.

Por lo tanto, el liderazgo puede ejercerse de diversas maneras. Emerge de la interacción y de la actividad en el grupo. Según Romero (1992: 28) "en todo grupo existen dos tipos fundamentales de actividad; el comportamiento intelectual y las conductas afectivas. La tarea que implica actividades racionales, intelectuales, requerirá de un liderazgo instrumental. Al mismo tiempo, la interacción entre los integrantes implica una actividad emocional. Existirá en un grupo también el liderazgo orientado hacia el mantenimiento afectivo del grupo".

El liderazgo intelectual encarará los problemas de logro grupal, mientras el segundo tipo de liderazgo se relaciona con el proceso grupal.

Le distinguen tres principales estilos de liderazgo: autoritario, autocrático, democrático y laissez faire.

El líder autoritario o autocrático trata a los miembros de un grupo como si fueran inferiores a él o ella, desestima sus ideas, ignora sus criterios y aportes. No escucha, tiende a mostrar trato preferencial hacia algunos miembros. Estas actitudes no son convenientes para el progreso del grupo.

El líder autocrático provoca dos tipos de reacciones: agresividad o apatía. El tipo de reacción que sobrevenga está relacionada con la dependencia del grupo con el líder. Reacción agresiva implica rebeldía hacia el líder y amistad entre los miembros del grupo (Chibás, 1992, p.20). El líder autoritario genera frustración 
entre los miembros. Puede surgir el rol de "chivo emisario", en el cual todo lo malo que sucede en el grupo se deposita en uno de sus integrantes.

De acuerdo con Maisonnuve (1985, p.31), el liderazgo democrático promueve las relaciones entre sus integrantes basadas en solidaridad, aparecen diferencias personales, amistad entre los miembros de grupo, existe mayor compromiso y gratificación en la realización de la tarea.

El líder democrático favorece las discusiones, la tensión es menor, presenta los pasos necesarios para alcanzar la tarea y los propone a discutir, no asigna tareas, ni forma subgrupos de trabajo, deja que éstos emerjan, busca el consenso.

El líder laissez faire constituye una especie de abandono de autoridad (Maisonnuve, 1985), no se interesa por la actividad de grupo, se deja desbordar por él, otorga recompensas y castigos en forma arbitraria, no se compromete con el grupo, solo está presente para que el grupo pueda recurrir a él en caso que lo necesite.

En un liderazgo laissez faire encontramos, según Romero (1992: 31), "falta de incentivo y ausencia de técnicas necesarias para arribar a decisiones y al planteamiento cooperativo, a menos que aparezca un líder endogrupal, de tipo democrático o autoritario, las fuerzas tendientes a la dispersión del grupo son cada vez mayores".

Para finalizar el análisis sobre los estilos de liderazgo es necesario aclarar, que el coordinador en un grupo tiene un rol fijo, diferenciado de los otros y funciones específicas.

A continuación, analizaré las estructuras de comunicación y el liderazgo. Líder es un miembro del grupo, que no siempre es líder formalmente asignado por la institución, sino un miembro que tiene "la capacidad de procesar la información reteniéndola o transmitiéndola" (Maisonnuve, 1985, p.33).

Partiendo de los planteamientos de Maisonnuve, al existir posiciones diferenciadas entre sus miembros, aquellos que se comunican con mayor frecuencia tienen a dirigirse palabras unos a otros y apoyarse entre sí. Son considerados como los más productivos y ocupan posiciones centrales. Los participantes alejados ocupan posiciones de bajo status y tienden a inhibirse en sus aportes.

Dado que los coordinadores ocupan un rol importante en los grupos de trabajo, es necesario tener presente errores en los cuales pueden caer. Chibás (1992: 46-47) señala algunos de ellos:

- "La carencia de una postura de adaptación a las características propias del grupo. 
- La necesidad de mostrar superioridad que origina el bloqueo en el grupo desde sus comienzos.

- La falta de seguridad en sí mismo y en sus conocimientos, que puede manifestarse mediante el uso de vocabulario difícil de entender. Esto puede llevar a que nadie se atreva a expresar sus ideas y se produzca un rechazo general del grupo hacia el coordinador.

- Trata de imponer sus ideas; esto ocurre cuando el coordinador inicia y termina la sesión; así induce al grupo a pensar de una determinada manera.

- El hablar demasiado y no escuchar a otros miembros del grupo impide que otros participantes se expresen. Esto puede ocasionar malestar en el grupo, pudiéndose pensar que el coordinador tiene mucho temor a los silencios, los cuales pueden ser provechosos pues denotan que los miembros del grupo están reflexionando sobre el tópico que se trata durante la sesión.

- El laxismo se manifiesta cuando quien coordina propone un método de trabajo y se deja llevar por los participantes. Esto pasa también cuando el coordinador llega a la sesión sin preparación previa.

- El exceso de autoridad genera bloqueos y resistencias en el grupo.

- La falta de autoridad puede traer la confusión. Con frecuencia éstas dos actitudes pueden coexistir en el coordinador. Este coordinador no llamará la atención de los miembros de mayor status cuando no cumplen con las tareas asignadas, sin embargo lo hará con un participante "débil".

- Excesiva reactividad surge cuando quien coordina es parte interesada en el asunto y muestra una tendencia a intervenir a favoro en contra de los participantes.

- El miedo al grupo se manifiesta en un coordinador que tiene actitudes que oscilan entre autoritarismo extremo, la agresividad muy sutil o la manipulación, hasta puede renunciar a la coordinación en provecho de un líder informal. Esta falta de seguridad se manifiesta en su conducta: se sienta "tieso" en su silla, mueve nerviosamente el pie o el bolígrafo, evita la mirada de los participantes.

- La falta de preparación de las sesiones sucede cuando el coordinador asiste a la sesión sin una propuesta de trabajo, sin tomar en cuenta decisiones que se han tomado en la sesión anterior.

- La falta de comprensión de la dinámica de grupo se produce cuando no se interpretan adecuadamente las conductas y las actitudes manifiestas o latentes.

- La pérdida de la coordinación se produce cuando uno o más miembros del grupo comienzan a orientarlo, guiarlo y los demás miembros del grupo a prestar más atención a éstos que al coordinador. El coordinador ya no es dueño de sí y de la situación y deja de conducir los procesos grupales. Esto suele sucederle a coordinadores inexpertos". 


\section{Encuadre}

Por encuadre entenderé "un conjunto de estipulaciones que asignan el mínimo de interferencias a la tarea, a la vez que facilita el máximo de información. Incluye un conjunto de variables, que quedaran fijadas y son el marco en el cual se ubica el proceso", (Roth, 1995). Chibás (1992) definen el encuadre como "la delimitación clara y precisa de la tarea grupal y las normas de funcionamiento". De lo señalado se puede visualizar la importancia del encuadre para el trabajo grupal desde la primera sesión ya que, en gran medida, el éxito depende de él. Todo encuadre tiene la parte una fija, que consiste en definir con el grupo el día, la hora, el lugar, la duración y la frecuencia de los encuentros. Una vez obtenido este acuerdo se delimitan las responsabilidades y las funciones tanto de la persona que coordina como de quienes integran el grupo.

Cuando el grupo y su coordinador llegan a un acuerdo sobre el encuadre, se está formalizando un contrato. El encuadre puede ser modificado siempre y cuando exista una justificación válida y ésta se explicita y discute en el grupo.

Otra parte del encuadre se refiere al proceso por el cual Roth $(1995, \mathrm{p} .2)$ entiende "la sucesión de eventos... acciones... que surgen en un espacio y durante un tiempo".

Chibás (1992, p. 41) sugiere tomar en cuenta otros elementos que deben formar parte del encuadre inicial de trabajo. Estos son:

- Clarificar la tarea.

- Establecer la metodología de trabajo.

- Definir los días en los cuales no se la sesionará y aclarar por qué.

- Aclarar el manejo de ausencias.

Se ha señalado, que es alrededor de la tarea que se organiza un grupo y por lo tanto, después de establecer un encuadre claro, los integrantes de un grupo comienzan a relacionarse con su coordinador, con la tarea y entre sí. Esto da inicio al surgimiento de roles relacionados con la tarea, con el mantenimiento del grupo o comienzan surgir roles individuales, que pueden obstaculizar el abordaje de la tarea institucionalmente asignada.

\section{Roles}

El rol, según Pichón-Riviere (Fumagalli, 1987: 3), "es un modelo organizado de conducta, relativo a una cierta posición del individuo en una red de interacción, ligado a expectativas propias y de los otros"... "es un instrumento de interacción 
que incluye conductas que por un lado, tienen que ver con nuestra personalidad y por otro lado, con lo que pasa en nuestro vínculo con otros y lo que pasa en un contexto más amplio, en la sociedad en que vivimos".

Siguiendo a Fumagalli (1987: 6) "Ios roles no surgen solamente de un aspecto intelectual, también en un rol ponemos en juego aspectos emocionales que nos sirven para vincularnos con el otro para resolver la tarea".

Pichón-Riviere (Fumagalli, 1987: 7) reconoce cuatro roles fundamentales, Estos son: el rol del líder, como alguien que define y orienta la acción; otro rol es el de portavoz. El portavoz denuncia algo y así pone en manifiesto una situación compartida por el grupo.

Generalmente denuncia un problema. El tercer rol de importancia en un grupo es el de chivo emisario. No es un rol cómodo, pero aparece "porque es el modo en que el grupo puede ubicar el problema" (Fumagalli, 1987: 8). El cuarto rol es el de saboteador, el que "hace la resistencia al cambio" (Fumagalli, 1987: 8).

Chibás (1992: 5) y Kenneth, Sheats (1996: 2-9) identifican los siguientes roles que pueden asumir los integrantes en un grupo con relación a la tarea.

\section{Roles relacionados con la tarea}

El iniciador presenta nuevas ideas o reconsidera la meta grupal, sugiere soluciones y procedimientos. Este rol es muy oportuno en el inicio de trabajo de un grupo.

El investigador busca información, sugiere ideas. La intervención del miembro que asume este rol es positiva en todas las etapas de trabajo grupal.

El informante presenta hechos $u$ opiniones generalizadas por las autoridades. Sus intervenciones son oportunas en la etapa de evaluación.

El inquiridor de opiniones pregunta para aclarar dudas sobre el trabajo que el grupo me está realizando. Las intervenciones del miembro que asume éste rol son oportunas durante la etapa de evaluación.

El opinante expresa su opinión o creencia relativa a una sugerencia o alternativa grupal. En la primera fase del grupo, no es oportuna la participación de este rol; en la fase de evaluación, la aparición de un miembro opinante es muy beneficiosa.

El elaborador cita ejemplos, elabora significados. Resulta útil en la primera fase.

El coordinador clarifica ideas y sugerencias de otros, trata de reunirlas, coordina actividades de los diferentes miembros o subgrupos. Resulta muy importante desde el inicio del trabajo en grupo. 
El orientador define la posición del grupo con respecto a la tarea, resume lo ocurrido, señala las divergencias entre las metas acordadas y cuestiona la dirección que está tomando el grupo. El rol del orientador es muy útil desde el inicio.

El dinamizador incita al grupo a la acción o a la toma de decisiones. Es muy conveniente su aparición desde la primera fase.

El técnico de procedimientos realiza tareas rutinarias como la de distribuir materiales, traer material audiovisual, fotocopias, entre otros. Es útil desde el inicio.

El registrador anota las sugerencias, las decisiones, acuerdos, o resultados de las discusiones. Este rol es muy provechoso en todas las fases del trabajo grupal.

Una de las funciones de la coordinación es fomentar la aparición de estos roles como también promoverlos relacionarlos con el mantenimiento del grupo.

\section{Roles relacionados con el mantenimiento del grupo}

El objetivo principal de estos roles es conservar el grupo y fortalecerlo.

El estimulador elogia, fomenta la participación de los integrantes, comprende sus puntos de vista, acepta opiniones de otros.

El conciliador interviene en disputas internas, mediando las diferencias entre los miembros, ofreciendo arreglos cuando hay conflicto.

El mediador o transigente actúa en situaciones de conflicto donde su idea o posición están involucradas, ofrece soluciones disminuyendo su status o admitiendo su error. Su aparición no es conveniente al inicio, pero sí durante la fase de evaluación.

El guardaagujas fomenta la participación de otros, intenta mantener abiertos los canales de comunicación, ofrece propuestas sobre cómo regular la comunicación.

El legislador lleva registro de diferentes aspectos del proceso grupal y hace comentarios al grupo sobre sus procedimientos.

El seguidor sigue el movimiento del grupo y es un oyente cordial; acepta pasivamente ideas de los otros, se adapta a la voluntad grupal.

\section{Roles individuales}

Estos roles los asumen algunos miembros en un grupo para resolver sus necesidades individuales. Su objetivo no se relaciona con la tarea grupal ni con el funcionamiento del grupo. La persona que coordina debe ponerles atención y tratar de disminuir su efecto sobre el grupo. Si interfieren con la tarea grupal, el coordinador puede interpretar su conducta para que el integrante tome conciencia de su rol y del efecto que éste tiene para alcanzar la tarea grupal. 
Al finalizar la reflexión acerca del trabajo en equipo, se puede concluir que un equipo se constrnye en un proceso crya duración es muy particular, ya que depende de la estructura de la personalidad de cada uno de sus integrantes y los niveles de identificación con la tarea por realizar
El agresor expresa la desaprobación por las acciones, los valores y los sentimientos de otros.

El obstructor siempre está en desacuerdo, se opone sin razones, es terco tiene actitud negativa, es resistente al cambio.

Al buscador de reconocimiento le gusta llamar la atención hablando de sus éxitos y logros personales.

El confesante busca la oportunidad para expresar sus sentimientos, intuiciones, ideologías pero sin interés para el grupo.

Elmundano ofrívolo demuestra la indiferencia hacia los trabajos del grupo, es cínico y descarado.

El dominador trata de afirmar su autoridad manipulando al grupo o alguno de sus miembros, sin interés en el estado en que se encuentra la tarea.

El buscador de ayuda trata de despertar la simpatía en los demás manifestando su inseguridad y confusión.

Al tomar en cuenta diferentes momentos por los cuales atraviesa un grupo en el proceso de conformar el equipo de trabajo, sería importante repensar cuáles roles debería, imprescindiblemente, fomentar la coordinación para facilitar la tarea previamente asignada. ¿Será posible evitar que surjan roles individuales? ¿Cómo se pueden disminuir los efectos de éstos en un grupo?

Al finalizar la reflexión acerca del trabajo en equipo, se puede concluir que un equipo se construye en un proceso cuya duración es muy particular, ya que depende de la estructura de la personalidad de cada unó de sus integrantes y los niveles de identificación con la tarea por realizar. También depende de la identificación con la coordina-ción así como de los integrantes entre sí y con la institución para la cual laboran.

"El trabajo en equipo responde siempre a una concepción que tiene el hombre acerca del mundo y acerca de las relaciones humanas" así lo señalan Beller y Mannigot (1987: 6) en su artículo acerca del trabajo en equipo.

Se puede afirmar que, constituir un equipo de trabajo surge durante un proceso en el que hay que tomar en cuenta varios elementos. Entre ellos cabe destacar la tarea alrededor de la cual el grupo se reúne y organiza. Otro aspecto de vital importancia es el encuadre inicial de trabajo, donde se debe establecer una clara estrategia metodológica, definir el espacio y tiempo de duración del trabajo, como también las reglas de funcionamiento y formas de comunicación de quien se encarga de coordinar con quienes integran el equipo, marquen las pautas de relaciones interpersonales que favorezcan o no el buen funcionamiento, productividad y un determinado clima grupal. 


\section{Referencias}

Ansieu, D. (1994). Los roles y las actitudes en los grupos pequeños y reducidos. (Sin datos).

Barreiro, T. (1993). Trabajos en grupo. Buenos Aires: Editorial Kapeluz.

Bauleo, A. y De Brassi (1990). Clínica grupal, clínica institucional. Madrid: Editorial Atvel S.M.

Beller, D. y Mannigot M. (1997). "Equipos de trabajo". En el periódico Uno y otros. Año III.

Bleger, J. (1961). Grupos operativos en la enseñanza. Conferencia dictada en la Asociación de Psicología y Psicoterapia de Grupo. Buenos Aires, Argentina.

Chaves, L. (1998). Introducción a la obra de Enrique Pichón-Riviere. (Sin datos).

Chibás, F. (1992). Creatividad, más dinámica de grupo. La Habana: Editorial Pueblo y Educación.

Fainstein, (1997). La moda de trabajar en equipo o el modo de trabajar en equipo. Buenos Aires: Universidad Buenos Aires.

Fainstein, (1997). ¿Qué son los equipos de Trabajo?. Buenos Aires: Universidad de Buenos Aires.

Fumagalli, C. (1987). Teoría del rol. Buenos Aires: Ediciones Cinco.

Kenneth, D. y Sheats B. (1996). Roles funcionales de los miembros de un grupo. Buenos Aires: Editorial Nueva Visión.

Lagarde, M. (1994). "Género en desarrollo". Memorias del Taller, ofrecido por la autora en Managua, Nicaragua.

López, F. (2000). Interdisciplinariedad, cultura organizacional y equipos académicos. Seminario "Capacitación para el Desarrollo Regional Sostenible", Heredia, Costa Rica. 
Maisonnueve, J. (1985). La dinámica de los grupos. Buenos Aires: Editorial Nueva Visión.

Podcaminsky, M. y Beausset, L. (1999). Aproximación a una comprensión de los grupos. San José: Contexto grupal.

Quiroga, A. (1980). Esquema conceptual, referencial y operativo (ECRO), base recolectada en la Primera Escuela de Psicología Social, Buenos Aires.

Romero, R. (1992). Grupo, objeto y teoría. Buenos Aires: Argentina.

Roth, M. (1995). Encuadre y proceso. Nociones teóricas y su importancia en la coordinación de grupos. San José: Contexto grupal.

Rud, C. (1999). Empatía, una manera de esta. Memoria del IX Encuentro Latinoamericano de Enfoque centrado en la persona, San José: Costa Rica.

Shapira, M. (1991). Acerca de la intervención en grupo de supervisión. Buenos Aires: Editorial Nueva Visión. 